\title{
Long-term-Storage Performance Degradation Causes of Fuse Electronic Components
}

\author{
Yingwei WU \& Xinglin Qi \& Tieshan Zhao \\ Ordnance Engineering College, Shijiazhuang, Hebei, 050003, P.R. China
}

Bingfeng Yang

Academy of Military Transportation, Tianjin, 300161, P.R. China

ABSTRACT: The performance for fuse electronic components may have degradation after long-term-storage. In order to as-certain the components which its performance has degradation, two fuse electronic parts, which have been stored for 10 years, are chosen as samples for an accelerated test. It shows that the white noise from the resistance and the interfering noise between different resistances may lead the performance degradation. The zero drift due to the brokenness of bonding wire may magnify the noise voltage when under storage, and influence the reliability of the operational amplifier. The result indicates that the resistances and operational amplifiers should be concerned more when test fuse electronic device after a long storage.

KEYWORD: Electronic components; Performance degradation; Failure analysis

\section{INTRODUCTION}

The significant development for science and technology and the great change for mode of operation bring great improvement and extension to fuse function; meanwhile, electronic components are also used in the fuse system more and more. Takes the fuse electronic device as an example, it can change the continuous electrical impulse signal into the electrical signal, and mainly for signal amplification, shaping and filtering, and then control the fuse by forming the control signal. The electronic device is composed of electronic components and its encapsulation materials, and the circuit component is shown in figure 1.

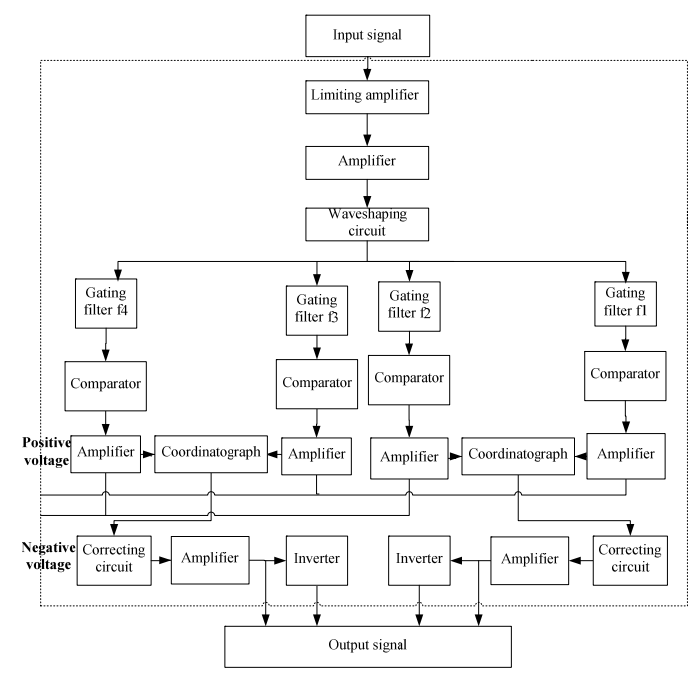

Fig. 1 Circuit diagram of electronic device
The electronic device plays an importance role in the fuse system, the fuse will not initiate warhead reliably, and even lead the whole ammunition system to failure when it is break down. However, there is little research directed against fuse electronic device function degradation or inefficacy reason at present. Therefore, we carry out accelerated degradation test based on two fuse electronic components which have been stored for 10 years, thus position the components which are easy to be degradation or failure, and its reason is also be analyzed.

\section{FAILURE MODE PREDICTION AND PERFORMANCE TEST}

\subsection{Failure mode prediction}

The circuit channels of electronic components are all composed of resistances, triodes and operational amplifier, they all belong to the electronic elements, although there are some differences in failure mode, but the failure reasons are the same, the number of electronic elements in different circuit is different, so the failure may occur in any part. Analyzing the failure mode of electronic components according to FMEA, the failure mode can be summarized as follows:

(1)In the process of signal amplification, shaping circuit may occur failure, therefore, the output signal amplitude may be low or no; 
(2)The voltage transmission without filtering makes the output voltage high when the filter circuit failure occurs;

(3)Calibration or calibration circuit failure will cause the output voltage be difficult to meet the requirements.

\subsection{Performance test}

According to the failure mode prediction, choosing testing project targeted. Then test its performance automatically by The Electronic Component TestBed, and get its performance degradation data. Specific testing items and testing positions are shown in table 1.

Tab. 1 Detection items and their target of electronic device

\begin{tabular}{|c|c|c|}
\hline Test Items & Implication & Test Positions \\
\hline $\begin{array}{l}\text { Positive/Negative } \\
\text { consumption current }\end{array}$ & $\begin{array}{l}\text { The current value consumed for the electronic components in circuit under the } \\
\text { effect of positive/negative voltage. }\end{array}$ & Connection between plates. \\
\hline Zero drift & $\begin{array}{l}\text { The output voltage of electronic components with only power but no input } \\
\text { signal. }\end{array}$ & Each operational amplifier. \\
\hline Transmission factor & The ratio of output voltage and input frequency. & $\begin{array}{l}\text { Band-pass circuit operational } \\
\text { amplifier. }\end{array}$ \\
\hline Impulse amplitude & $\begin{array}{l}\text { The different value between the maximum and the minimum of output voltage } \\
\text { under the same input signal. }\end{array}$ & $\begin{array}{l}\text { Low-pass filter circuit operational } \\
\text { amplifier. }\end{array}$ \\
\hline Output voltage different & The different value between output voltages under the different input signal. & Capacitance in shaping circuit. \\
\hline Output phase different & The different value between output phases under the different input signal. & Capacitance in limiting amplifier \\
\hline
\end{tabular}

\section{ACCELERATED DEGRADATION TEST RESULTS AND FAILURE REASON ANALYSIS}

\subsection{Test results}

Accelerated degradation test must comply with the premise of constant failure mechanism, it can predict the life characteristic of products on normal stress level based on the performance degradation data on high stress levels, thus effectively solve the problems of traditional reliability life test, including long time, low efficiency and high cost ${ }^{[2]}$. In order to save time and cost for test, select two fuse electronic components that have been stored for 10 years to carry on accelerated degradation test, test the parameters regularly, including positive and negative consumption current $I$, zero drift $U_{O}$, transmission coefficient $k$, the pulsation amplitude $U_{F}$, the output voltage difference $U_{C}$, and the output phase difference $\varphi$.

The results show that $U_{F}, U_{C}, k$ and $\varphi$ of the two samples are all maintain in the stable level, and there is no obvious degradation trend. The changing trend of $I$ is shown in figure 2 and figure 3. The changing trend of $U_{O}$ is shown in figure 4 .

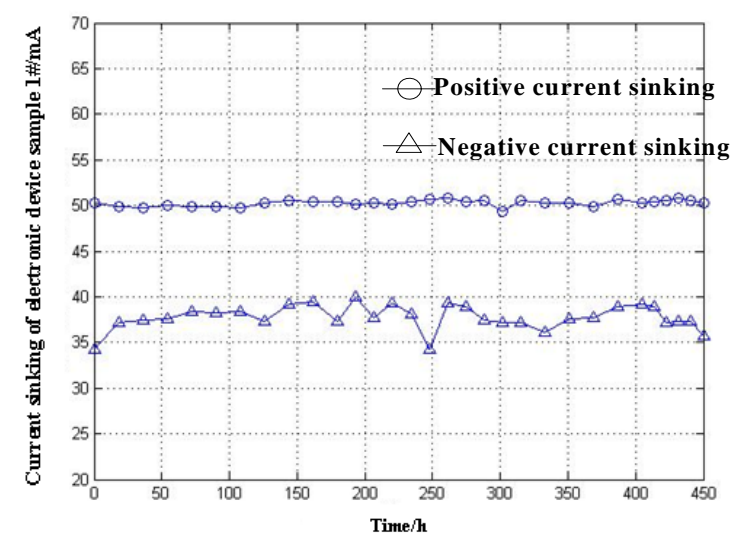

Fig.2 Current trend of electronic component sample 1\#

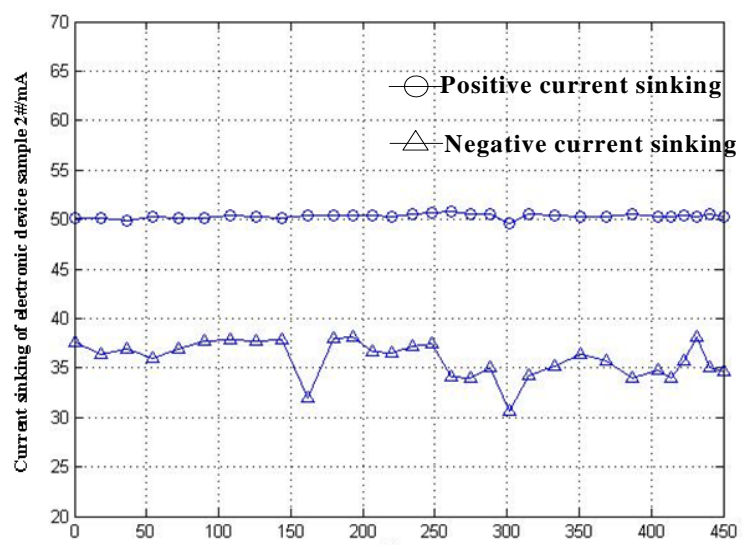

Fig. 3 Current trend of electronic component sample 2\# 


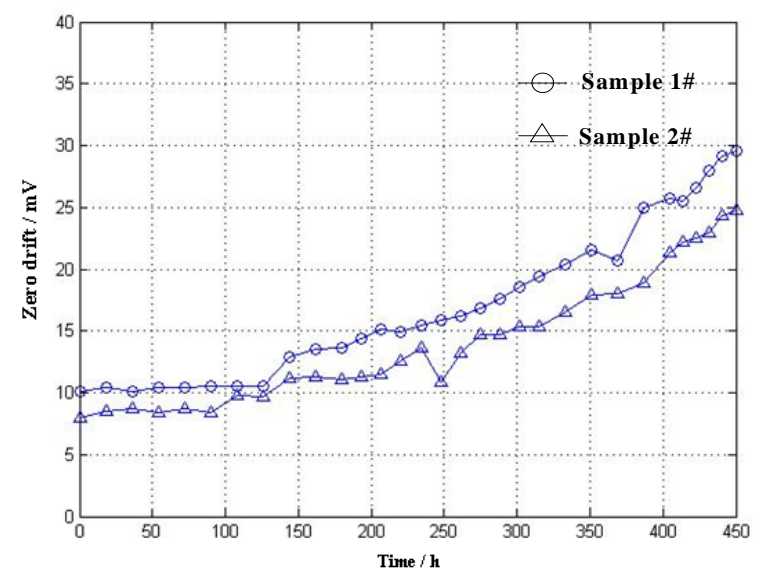

Fig. 4 Zero drift trend of electronic component sample

As is shown in figure 2 and figure 3, we can see that the changing rules for current between sample $1 \#$ and sample $2 \#$ are very similar. As for positive consumption current, both of the samples are stable in $50 \mathrm{~mA}$. For sample $1 \#$, the maximum is $50.841 \mathrm{~mA}$ and the minimum is $49.283 \mathrm{~mA}$; for sample $2 \#$, the maximum is $50.776 \mathrm{~mA}$ and the minimum is 49.613mA. As for negative consumption current, for sample 1\#, it fluctuates around $37.736 \mathrm{~mA}$, the minimum is $34.244 \mathrm{~mA}$ and the maximum is $40.034 \mathrm{~mA}$; for sample 2\#, it fluctuates around $35.877 \mathrm{~mA}$, the minimum is $30.621 \mathrm{~mA}$ and the maximum is $38.145 \mathrm{~mA}$, it can be seen that the fluctuation of negative consumption current is larger. We can see from figure 4 that the zero drift of two samples both change obviously, the zero drift of electronic components has obvious degradation trend over time.

Accelerated degradation test can truly reflect the real degradation mechanism only under the invariance of degradation mechanism. Therefore, checking the invariance of degradation mechanism is important. The rate of degradation for zero drift under the temperature stress can be expressed with Arrhenius equation as:

$\frac{d M}{d t}=A \exp \left(-\frac{Q}{k T}\right)$

In the formula: $M$ represents the rate of degradation; $Q$ represents the activation ability; $A$ represents the constant; $E$ represents activation energy; $k$ represents the Boltzmann Constant; and $T$ is served as absolute temperature.

$Q$ keeps unchangeable when the degradation mechanism is consistent. From formula (1), when the failure mechanism is unchangeable, there is linear relation between $\ln (d M / d t)$ and $-1 / T$. Therefore, once we can ensure the linear relation, we can say that $Q$ is consistent, namely that the failure mechanism is unchangeable. The relation between $\ln (d M / d t)$ and $-1 / T$ of zero drift for two samples is shown in figure 5.

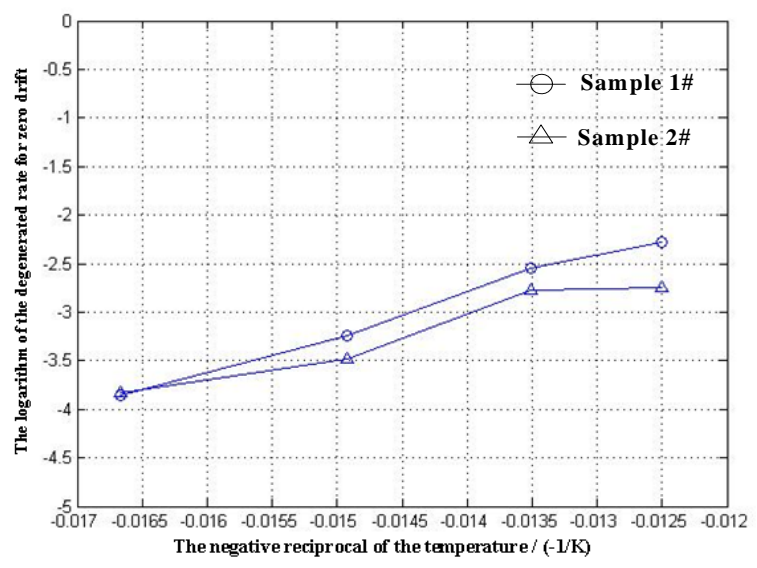

Fig. 5 Relation between $-1 / T$ and $\operatorname{In}(d M / d t)$ of zero drift

Knowable by figure 5 , it is linear relation between $-1 / T$ and $\ln (d M / d t)$ of zero drift, we can define that $Q$ keeps unchangeable, namely that the failure mechanism is consistent.4 Design and Optimization for Planar-Type Voice Coil Motor

\subsection{Failure reason analysis}

The zero drift is decided by the entire specific performance of the electronic circuit, it due to the noise voltage in circuit, and relate to every component in the circuit, the resistances themselves have white noise, they can also produce interference noise between each other; many operational amplifiers distribute in every circuit, and one noise produced by a component can pass through the circuit and the next circuits, thus it would be magnified for many times. After long storage, the electronic components would be aging, and then increase the noise, because the number of electronic components is large and the degradation can be accumulated, the degradation trend will be more evidently. Next, let's analyze the performance degradation mechanism of the resistances and operational amplifiers.

(1)Resistance's Failure Mechanism Analysis:

The white noise exists because of the interaction between the free electrons and the vibration ions ${ }^{[5]}$, its power can be obtained by Johnson Formula: $P=k \times T \times B W$

Using the square of the source voltage to express the thermal voltage as:

$e^{2}=4 k T R \times B W$

In the formula: $P$ is for noise power; $k$ is for Boltzmann Constant; $T$ is for the temperature; $B W$ is for the system bandwidth; $e^{2}$ is for the thermal voltage; and $R$ is for the resistance.

Resistance will be enlarged after long storage and knowable by formula (3), the thermal voltage would increase along with the resistance's enlargement, thus make the thermal noise to be bigger, and it will be more obviously when the temperature is higher. 
(2)Operational Amplifier Failure Mechanism Analysis:

The operational amplifier chip are bonding together with the base by the conducting resin, the conducting resin's performance degradation exists when it is under storage, then the conducting resin's bonding performance may degenerate or even lose, thus the partially or completely separation may happen between the operational amplifier chip and the base, and the bonding wire could disconnect when suffered from external mechanical forces ${ }^{[3][7]}$. And then zero drift would exist, zero drift will be greater and the degradation will be more obviously over time.

\section{CONCLUSIONS}

This paper chooses two fuse electronic components which have been stored for 10 years to carry out the accelerated degradation test, check the parameters regularly, which include positive and negative consumption current $I$, zero drift $U_{O}$, transmission coefficient $k$, the pulsation amplitude $U_{F}$, the output voltage difference $U_{C}$, and the output phase difference $\varphi$. It shows that the changing rules for positive and negative consumption current are very similar, but the fluctuation of the negative consumption current is bigger. Over time, the degenerating trends of zero drift for electronic components become more apparent. The other parameters are relatively stable, indicating that the design for the entire circuit is rational, though they may be aging after long storage, the performance for the whole circuit is still good.

It indicates that the resistance's white noise for itself may give the degradation by further analysis; we can also know that the bonding wire would disconnect after long storage, bring about degradation for the operational amplifier, and even influence the fuse reliability. Consequently, more attention should paid to the resistance and an operational amplifier when test the fuse electronic components after long storage.

\section{REFERENCES}

DENG. A. M. 2006. Research on Reliability Technology of High-reliability and Long-lifetime Products. Changsha: National University of Defense Technology: 54-58.

JIANG T. M. \& WANG X. H. 2012. Reliability and Life Test. Beijing: National Defense Industry Press.

Vona S A, Tong J R Q K, Kuder R, et al. 1998. Surface Mount Conductive Adhesives with Superior Impact Resistance. International Symposium on Advanced Packaging Materials: 261-267.

WANG J. \& WENG L. \& ZHANG H. 2010. The Accelerated Testing to Estimate the Reliability Specifications. Electronic Product Reliability and Environmental Testing, 28(4): 6-10.
WU G. \& ZHANG X. P. 2006. Research of Resistance Thermal Noise. Ordnance Industry Automation, 25(4): 8687.

ZHAO W. \& YANG. J. 2012. Evaluation on Storage Reliability of Pyrotechnics Based on Performance Degradation Data. Chinese Journal of Energetic Material, 20(4): 406-408.

Zwolinski M, Hickman J, et al. 1996. Electrically conductive adhesives for surface mount solder replacement. IEEE Transactions on Components, Packaging and Manufacturing Technologies, 19: 241-250. 